msh-mss Mathématiques et sciences humaines

155-156 | Automne-Hiver 2001

Varia

\title{
L'argumentation statistique dans la politique sociale
}

Statistical argumentation in social policy

\section{Thierry Foucart}

\section{(2) OpenEdition \\ 12 Journals}

Édition électronique

URL : http://journals.openedition.org/msh/2853

DOI : $10.4000 / \mathrm{msh} .2853$

ISSN : 1950-6821

Éditeur

Centre d'analyse et de mathématique sociales de l'EHESS

Édition imprimée

Date de publication : 1 décembre 2001

ISSN : 0987-6936

\section{Référence électronique}

Thierry Foucart, "L'argumentation statistique dans la politique sociale », Mathématiques et sciences humaines [En ligne], 155-156 | Automne-Hiver 2001, mis en ligne le 10 février 2006, consulté le 23

juillet 2020. URL : http://journals.openedition.org/msh/2853 ; DOI : https://doi.org/10.4000/msh.2853

〔c École des hautes études en sciences sociales 
Math. \& Sci. hum., (39 année, ${ }^{\circ} 156,2001$, p. 33-42)

\title{
L'ARGUMENTATION STATISTIQUE DANS LA POLITIQUE SOCIALE
}

\author{
Thierry FOUCART ${ }^{1}$
}

RÉSUMÉ - Les règles établies à partir d'études statistiques ne garantissent pas le respect des intérêts individuels, et leur application systématique peut même aboutir à un résultat globalement négatif. Après avoir montré cette ambiguïté dans le cas particulier du modèle linéaire et donné un exemple très simple, les politiques menées actuellement dans l'éducation nationale et la santé publique sont justifiées par des analyses statistiques en fait discutables. Elles ont pour conséquence l'application à chacun de règles collectives, parfois à l'encontre de l'intérêt individuel.

MOTS-CLÉS - Modèle linéaire, Intervalle de prévision, Règle de décision, Prévision, Utilité.

SUMMARY - Statistical argumentation in social policy

The rules laid down from statistical studies do not guarantee the respect of individual interest, and their systematic application can even lead to an overall negative result. After showing this ambiguity in the particular case of the linear model and giving a straightforward example, the politics in national education and public health are justified by statistical analyses which are debatable in fact. Their consequence is the application to each one of collective rules, sometimes against individual interest.

KEYWORDS - Linear model, Prevision interval, Decision rule, Prevision, Utility.

\section{INTRODUCTION}

L'application systématique d'une règle établie à partir de statistiques ne garantit pas toujours un résultat satisfaisant pour deux raisons :

- la première est la difficulté d'interprétation des statistiques ${ }^{2}$;

- la seconde est plus technique : les critères utilisés en statistiques ne coïncident pas avec la notion d'utilité fondamentale en sociologie ${ }^{3}$ et les modèles sont parfois appliqués sans que les conditions nécessaires à leur validité soient vérifiées.

C'est ce dernier point qui est développé ici, en proposant tout d'abord un exemple élémentaire d'application maladroite des résultats d'un modèle linéaire. Ensuite, on

\footnotetext{
${ }^{1}$ Maître de Conférences à l’Université de Poitiers, e-mail : tfoucart@wanadoo.fr

2 Thierry Foucart, «L’interprétation statistique », Mathématiques et Sciences humaines, n 153, 2001, p. 21-28 ou Stephen Jay Gould, La mal-mesure de l'homme, Odile Jacob, 1996, p. 278-297, en particulier.

${ }^{3}$ J. Rawls, Théorie de la justice, 1987.
} 
montre que les politiques sociales de l'Éducation Nationale et de la santé publique fonctionnent sur un schéma analogue, dont les effets pervers se font largement sentir par des usagers.

\section{DU MODÈLE EXPLICATIF AU MODÈLE PRÉVISIONNEL}

Le modèle linéaire consiste à représenter une relation entre une variable expliquée (ou dépendante) $Y$ (par exemple le salaire), et $p$ variables explicatives (ou indépendantes) $X_{j}, j=1, \ldots, p$ (par exemple la durée du travail, l'âge, la qualification, le secteur d'activité), par l'équation ci-dessous :

$$
Y=\square_{0}+\square_{1} X_{1}+\square_{2} X_{2}+\ldots+\square_{j} X_{j}+\ldots+\square_{p} X_{p}+\square
$$

dans laquelle :

- les coefficients $\square_{j}$ sont des paramètres théoriques appelés coefficients de régression ;

- la variable $\square$ est une variable aléatoire centrée appelée variable résiduelle ;

- on suppose fréquemment que la variable $\square$ suit la loi normale $N(0, \square)$.

L'estimation des paramètres du modèle est effectuée de façon classique sur un ensemble de $\mathrm{n}$ observations des variables $Y$ et $X_{j}:\left(y^{i}, x_{j}^{i}\right), i=1, \ldots n$ suivant le critère des moindres carrés.

Le modèle linéaire est utilisé principalement :

- dans un objectif de description : il s'agit alors de décrire les relations entre les variables explicatives et la variable expliquée ;

- dans un objectif de prévision : la formule est appliquée à d'autres valeurs $x_{j}^{i}$ des variables explicatives pour prévoir la variable expliquée correspondante $y^{i}$.

La démarche descriptive n'est pas toujours facile : on retrouve ici des difficultés dont il a déjà été question dans le précédent article déjà cité. Elle consiste à analyser les relations en examinant les coefficients de corrélation partielle, qui mesurent la liaison entre la variable expliquée et une variable explicative après élimination de l'effet des autres variables explicatives sur cette liaison ${ }^{4}$.

Pour un néophyte, les résultats sont parfois surprenants : par exemple, une corrélation faible entre la variable expliquée et une variable explicative ne contredit pas toujours l'existence d'une relation linéaire significative conditionnellement aux autres, une corrélation forte peut devenir faible si l'on tient compte d'une autre variable explicative...

Cela peut conduire à des interprétations erronées, parfois même fondées sur des motivations personnelles plus que sur des déductions scientifiques : les analyses des

\footnotetext{
${ }^{4}$ On trouvera un exemple développé dans T. Foucart, L'analyse des données mode d'emploi, Presses Universitaires de Rennes, 1997, p. 130.
} 
résultats obtenus par des régressions multiples proposées dans l'ouvrage The Bell Curve de C. Murray et R. Herrnstein sont ainsi fortement critiquées par Stephen Jay Gould (op. cit., p. 392).

Mais c'est dans la démarche prévisionnelle que le modèle est utilisé de façon abusive dans certains cas.

Cette démarche prévisionnelle consiste à estimer l'espérance conditionnelle $E(Y / x)$ de $Y$ pour des valeurs $x_{j}, j=1, \ldots, p$ fixées des variables explicatives. L'estimation $m(y / x)$ est obtenue en remplaçant dans l'équation du modèle les coefficients de régression théoriques $\square_{j}, j=0, \ldots, p$ par leurs estimations $b_{j}, j=0, \ldots, p$ et en posant :

$$
m(y / x)=b_{0}+b_{1} x_{1}+b_{2} x_{2}+\ldots+b_{p} x_{p}
$$

Cette moyenne conditionnelle est utilisée comme approximation des valeurs individuelles de $Y$ pour les valeurs $x_{j}, j=1, \ldots, p$ fixées. On la complète par un intervalle de prévision dont la probabilité $p$, ou niveau de confiance, est fixée (en général à 0.95 ) et déterminée à partir de la propriété suivante, valable dans des conditions assez générales.

La loi conditionnelle de la variable $T=(Y-E(Y / x)) / S_{x}$ pour les valeurs $x_{j} j=1, \ldots, p$ fixées est la loi de Student de degré de liberté $n-p-1, n$ étant le nombre d'observations, $p$ le nombre de variables explicatives et $S_{x}{ }^{2}$ l'estimateur sans biais de la variance conditionnelle de $Y^{5}$.

Si le nombre d'observations est suffisant pour que la loi de Student soit correctement approchée par la loi normale centrée réduite, la probabilité que $Y-E(Y / x)$ soit supérieure à $0,675 S_{x}$ en valeur absolue est égal à 0,5 :

$$
\left.P\left(\square Y-E(Y / x) \square S_{x}\right)>0,675\right)=0,5
$$

Par suite, si un écart entre les valeurs réelle et estimée supérieur ou égal à 0,675 $s_{x}$ n'est pas acceptable, l'attribution de la valeur $m(y / x)$ aux individus donne environ $50 \%$ d' "insatisfaits », c'est-à-dire d'individus ne satisfaisant pas à la condition d'acceptabilité posée. Inversement, on peut estimer le pourcentage d' « insatisfaits » pour un niveau fixé de l'acceptabilité de l'erreur.

Concrétisons ce développement par un exemple très simple : on effectue la régression de la taille (en $\mathrm{cm}$ ) par le poids (en $\mathrm{kg}$ ) d'un groupe de jeunes filles d'une vingtaine d'années (les données sont réelles). Le modèle estimé à partir de 90 observations est le suivant :

$$
\text { Taille } \square 0,3985 \square \text { Poids }+142,195
$$

\footnotetext{
${ }^{5}$ G. Saporta, Probabilités, Analyse de données et Statistique, Technip, 1990, p. 373 et 387.
} 
Les résultats statistiques sont très satisfaisants (la liaison est linéaire, les lois sont normales, le coefficient de détermination $R^{2}=0,159$ est hautement significatif, et la variance des résidus est égal à $\left.s^{2}=27,07\right)$.

Imaginons que l'on distribue des vêtements en fonction du poids, en appliquant la formule précédente, et que l'erreur sur la taille des jeunes filles soit acceptable si elle ne dépasse pas $3 \mathrm{~cm}$. Le calcul statistique montre que $58 \%$ environ des jeunes filles de 60 $\mathrm{kg}$ ne correspondent pas à la taille calculée $(166,11 \mathrm{~cm})$ : dans $58 \%$ des cas, on distribue des vêtements trop grands ou trop petits. Dans le cas le plus favorable, c'est-àdire lorsque le poids est égal au poids moyen $(55 \mathrm{~kg})$, ce pourcentage est égal à $43 \%$. La règle de décision adoptée ici ne donne visiblement pas un bon résultat.

En outre, la formule de prévision n'est valable que sur des données extraites de la population dont est issu l'échantillon utilisé pour effectuer les calculs : il est possible que la structure des données sur la taille et le poids des jeunes filles de 20 ans ait changé depuis 1975, date à laquelle les observations ont été effectuées (en particulier les moyennes). L'« insatisfaction » risque d'en être accentuée.

Malgré l'imprécision des prévisions, la tendance actuelle consiste à appliquer à chaque individu un modèle établi sur des moyennes. Cette procédure est couramment appliquée dans le domaine commercial. Par exemple, la classification de la clientèle d'une banque suivant des critères financiers, familiaux et personnels, par une analyse discriminante, peut avoir pour objectif de déterminer les conditions dans lesquelles la banque accorde un prêt. Dans les sociétés de ventes par correspondance, on cible les mailings pour améliorer le taux des réponses et diminuer les coûts d'affranchissement etc.

L'efficacité du modèle est due au caractère commercial de l'entreprise : une mauvaise prévision peut provoquer la perte d'un client, mais n'est pas susceptible de modifier une règle de décision rentable sur un grand nombre. L'entreprise ne se préoccupe de l'intérêt d'un client particulier que pour maximiser son bénéfice global, calculé sur la totalité de la clientèle, et mesuré par des calculs comptables vérifiés indépendamment par les commissaires aux comptes. Dans ce contexte, la prévision statistique est un outil de gestion efficace.

Dans les services publics, la situation est différente : les usagers ne sont pas des clients. L'objectif est d'améliorer le service pour tous, non de maximiser le bénéfice de l'institution. La fonction d'utilité n'est pas la même : dans le cas d'une entreprise, cette fonction est définie par le bénéfice, alors que pour les services publics, c'est un agrégat des satisfactions individuelles.

L'administration est chargée à la fois d'appliquer les décisions prises au niveau politique et d'en contrôler l'efficacité : sa soumission de fait au pouvoir politique la rend à la fois juge et partie. En outre, la politique actuelle, définie au niveau de l'Union Européenne, est plus de réduire les dépenses publiques que de satisfaire un ensemble de besoins individuels. 
En appliquant dans ce contexte une règle de décision fondée sur un modèle de prévision analogue à celui présenté dans le paragraphe précédent, on s'expose aux mêmes difficultés : cela ne garantit pas la satisfaction de la majorité des gens concernés et peut même avoir pour effet une insatisfaction majoritaire.

\section{DE L’USAGE DE LA STATISTIQUE DANS LA POLITIQUE DE L'ÉDUCATION}

La politique de l'Éducation Nationale est actuellement définie par la loi d'orientation du 10 juillet 1989, qui contient l'article suivant :

Article 3. - La Nation se fixe comme objectif de conduire d'ici dix ans
l'ensemble d'une classe d'âge au minimum au niveau du certificat d'aptitude
professionnelle ou du brevet d'études professionnelles et $80 \%$ au niveau du
baccalauréat.

Cette loi considère donc comme acquis le fait que les élèves possèdent les capacités correspondantes, et induit une politique éducative sous contrôle statistique. La fonction d'utilité est simplement définie par la loi, et ne prend en compte en aucune façon les intérêts individuels.

Dès lors, l'intervention de l'administration ne se limite plus au calcul budgétaire : au plan pédagogique, dans les lycées et collèges, l'appréciation des enseignants sur un élève a de moins en moins d'importance dans son orientation à l'inverse des normes administratives et pédagogiques collectives, le but fixé étant quantitatif.

Cette intervention n'est pas nouvelle : déjà dans les années 60, les instituteurs recevaient des instructions concernant la répartition des notes de leurs élèves qui devait ressembler à un chapeau de gendarme, c'est-à-dire à la loi normale (témoignage oral de M. Claude Benoist, directeur d'école en retraite, Nevers).

Cette démarche constitue une déviation fréquente de la statistique qui s'exprime de la façon suivante : on a constaté que les notes étaient réparties selon la loi normale, donc les notes données par chaque instituteur doivent vérifier cette répartition. La propriété constatée - la normalité de la distribution - devient un objectif à atteindre. Le modèle gaussien, déduit de l'ensemble des notes, est ainsi imposé au plan individuel : on ne s'étonnera pas qu'il soit vérifié par les nouvelles données, et par la suite biaisé. Ce biais est d'autant plus important que les notes ne sont pas toujours distribuées suivant la loi de Laplace Gauss en l'absence de contrainte.

Un autre exemple précis de cette intervention est donné par les décisions de redoublement. On trouve, dans la circulaire de rentrée publiée au BOEN du 9 janvier 1998 :

L'observation des résultats des conseils de classe en juin 1997 fait apparaître que le taux de redoublement en fin de sixième s'est accru en moyenne nationale de deux points, passant de 9,86 \% en 1996 à 11,96 \% en 1997, avec des évolutions très contrastées selon les lieux, pouvant dépasser $20 \%$ dans certains collèges. 
Une telle évolution n'est pas souhaitable. Des études ont été conduites sur les effets du redoublement de la sixième. Elles font apparaître généralement que ce redoublement ne constitue pas en lui-même un gage de succès et peut au contraire aboutir à une régression des acquis individuels et à une dévalorisation de l'image que les élèves se font d'eux-mêmes et de l'école.

Suite à cette circulaire, les principaux, les yeux fixés sur les statistiques et sous la surveillance des rectorats, limitent les redoublements. Les considérations pédagogiques collectives, déduites d'études statistiques antérieures (les cohortes d'élèves sont observées depuis 1989) et parfois étrangères (souvent américaines, faute d'études françaises) sont ainsi appliquées à chaque établissement et à chaque cas particulier. On substitue à une appréciation de chaque élève par les professeurs une appréciation fondée sur un objectif politique quantitatif et des analyses statistiques discutables puisqu'effectuées à partir d'autres cohortes qui ont subi elles-mêmes d'autres réformes. On ne se préoccupe plus de l'intérêt de chacun, mais d'un intérêt supposé général et on applique au niveau individuel des normes établies au plan national, sans prendre en compte l'acceptabilité des erreurs commises : l'analogie avec la distribution de vêtements montre le danger de cette démarche, et donne un éclairage particulier aux difficultés actuelles du système éducatif.

L'évaluation des réformes administratives du système éducatif est souvent avancée pour montrer leur bien-fondé. On peut regretter qu'elle soit effectuée par des études statistiques menées par l'auteur lui-même des réformes, c'est-à-dire le ministère.

On sait que $71 \%$ des élèves entrés en $6^{\mathrm{e}}$ en 1980 ont atteint la classe de $3^{\mathrm{e}}$, contre $82 \%$ (en $3^{\mathrm{e}}$ générale) des élèves entrés en $6^{\mathrm{e}}$ en 1989 (note d'information $\mathrm{n}^{\circ} 98.01 \mathrm{du}$ M.E.N.). Certains auteurs, par exemple P. Joutard, ancien recteur, et C. Thélot, ancien directeur de la prévision du ministère de l'Éducation Nationale, en déduisent une augmentation du niveau scolaire (Réussir l'école, pour une politique éducative, Le Seuil, 1999).

Or un critère non statistique d'appréciation du niveau scolaire serait de comparer les épreuves à quelques années d'intervalles. Voici une dictée de 1974 du brevet d'études du premier cycle (BEPC, fin de $3^{\mathrm{e}}$ ) de l'Académie de Paris :

Pendant toute la durée du spectacle, Svétlana douta de ses yeux et de son intelligence. Il y avait trop de merveilles à voir, et elles se succédaient à un rythme trop accéléré pour qu'il fût possible de les apprécier au passage. Des pitres au museau enfariné et aux tuniques éclaboussées d'étoiles déchaînaient le rire énorme de la foule par une explosion de claques, de coups de pied au derrière et de cabrioles élastiques. Puis c'étaient des rugissements de fauves, et un ours savant se tenait en équilibre sur une boule, tandis qu'autour de lui les fouets claquaient comme des pétards. Des ballerines en paillettes bleues chevauchaient de nobles coursiers aux crinières de soie, des adolescents aux maillots roses construisaient une pyramide de bras et de jambes qui s'écroulaient soudain, et ils se retrouvaient au complet, l'un à coté de l'autre, souriants, moustachus, avec 
des joues de porcelaine et des yeux de diamants. Et, de nouveau, accouraient les clowns dans leurs habits trop larges.

Henri TROYAT, Tant que la terre durera

(Écrire au tableau : Svétlana).

Et la dictée du brevet des collèges de l'année 2000 :

Pourtant, il avait un père et une mère. Mais son père ne pensait pas à lui et sa mère ne l'aimait point. C'était un de ces enfants dignes de pitié entre tous qui ont un père et une mère et qui sont orphelins. Il n'avait pas de gîte, pas de pain, pas de feu, pas d'amour : mais il était joyeux parce qu'il était libre.

Victor HUGO, Les Misérables

Sans même parler de la notation de cette dernière dictée, tournée en dérision par de nombreux enseignants et journalistes (Le Monde du 7 juillet 2000, Libération du 10 juillet 2000), il est bien évident qu'il ne s'agit pas de la même troisième, au moins en ce qui concerne les exigences en orthographe. Les statisticiens n'ont pas comparé le niveau scolaire des élèves de $3^{\mathrm{e}}$ mais seulement le pourcentage de réussite à deux examens différents. Affirmer à la lecture des statistiques que le niveau scolaire des élèves de $3^{\mathrm{e}} \mathrm{s}$ 'est élevé est une erreur, qui consiste à remplacer l'observation qualitative par un calcul statistique : l'observation qualitative, effectuée par un observateur, est subjective mais fondée sur une appréciation globale, tandis que la seconde ne présente qu'une objectivité apparente puisqu'elle ne tient pas compte de l'aspect qualitatif.

Ces statistiques ne mesurent en réalité que l'application de la loi d'orientation de 1989, qui prévoit que la totalité des élèves entrant en $6^{\mathrm{e}}$ doivent parvenir en $3^{\mathrm{e}}$ (toutes filières confondues, CAP et BEP).

\section{DE L’USAGE DE LA STATISTIQUE DANS LA POLITIQUE DE LA SANTÉ}

Les méthodes statistiques ont été utilisées au départ dans la santé publique pour contrôler de façon scientifique l'efficacité des médicaments et des traitements médicaux, en vue de leur remboursement par la sécurité sociale. Ces méthodes, utilisées aussi en agronomie, sont très classiques et donnent des résultats fiables lorsqu'elles sont correctement appliquées.

On assiste maintenant à la codification des pathologies, terminée en médecine somatique et en cours en médecine psychiatrique (c'est ce que l'on appelle le PMSI). Ces codifications permettent une gestion budgétaire des pathologies, en déterminant pour chacune d'entre elles un coût et une norme, en durée d'hospitalisation par exemple.

Par suite, la politique de santé publique peut, et c'est le cas actuellement, avoir comme objectif de maîtriser les dépenses de santé. Cette maîtrise est conçue de façon 
collective, les acteurs (médecins, kinésithérapeutes, ...) étant considérés comme responsables de l'augmentation des dépenses de santé, de même que les patients.

Un exemple de la problématique posée par une décision thérapeutique est donné par le traitement des cancers de la prostate :

La prostatectomie radicale [N.B. l'ablation de la prostate] est le seul traitement réellement curatif de la prostate à ce stade [cancer limité à la capsule prostatique, stade TO-2,NO, MOJ. Il paraît légitime chez un patient en bon état général, présentant une espérance de vie supérieure à 10 ans, en règle âgé de moins de 70 ans $^{6}$.

L'indication thérapeutique est ainsi fondée sur l'espérance de vie du patient, c'està-dire une moyenne. On peut s'inquiéter de la légitimité de cette règle quand on sait que le serment d'Hippocrate, texte fondateur de la déontologie médicale, impose au médecin de donner le meilleur traitement médical à son patient en dehors de toute autre considération, et que cette moyenne statistique n'a aucun sens au plan individuel. On retrouve la procédure d'estimation présentée dans le premier paragraphe: les informations prises en compte pour évaluer l'espérance de vie du patient (âge, état de santé général) jouent le rôle des variables explicatives, la variable expliquée étant le temps qui lui reste à vivre. L'application systématique de cette règle thérapeutique peut provoquer, comme cela a été expliqué précédemment, une multiplication des erreurs.

Un autre exemple de problématique est donné par le dépistage de la trisomie 21 chez les femmes enceintes. La règle proposée pour ce dépistage (en 1988) peut être interprétée en termes économiques et éthiques ${ }^{7}$ : on propose aux familles un dépistage lorsque le risque statistique d'une fausse couche d'un enfant normal provoquée par le geste médical (un prélèvement du liquide amniotique) est égal au risque d'une naissance d'un enfant trisomique, suivi d'une interruption de grossesse dans le cas d'une anomalie génétique du fotus. Le critère d'égalité des risques revient à considérer de facto que les deux situations sont équivalentes en termes de coût économique et humain, et on émet implicitement un jugement de valeur sur la vie d'un trisomique. C'est une démarche éthique collective (Moatti et coll.), mais il est bien difficile de savoir si la majorité des familles concernées partagent cette façon de décider : là encore, la règle de décision collective n'a pas grand-chose à voir avec les choix individuels.

La codification des pathologies et le calcul des coûts permettent de transformer les indications thérapeutiques en obligations administratives.

Dans les deux exemples précédents, cette transformation est impossible actuellement : ce serait de l'eugénisme trop voyant. On procède donc par incitation.

\footnotetext{
${ }^{6}$ E. Fontaine, Ph . Le Prise, P. Nehamia, D. Beurton, «Diagnostic et traitement actuel du cancer de la prostate », La Lettre Chirurgicale Européenne, février 1993, n¹55.

${ }^{7}$ J.-P. Moatti, C. Julian-Reynier, V. Séror, C. Le Gales, S. Aymé, «Evaluation économique et éthique médicale », Natures-Sciences-Sociétés, 1993, 1, 4.
} 
Dans le premier cas, cette dernière est faite par le biais de la contrainte budgétaire et de la réduction du nombre de lits. Le problème se pose aussi pour tous les traitements médicaux coûteux (médicaments, prothèses, etc....) : la contrainte budgétaire impose aux médecins de sélectionner les malades auxquels on donnera un pacemaker, qui seront opérés, auxquels on prescrira des médicaments coûteux etc. Dans le cas du cancer de la prostate, l'indication thérapeutique est fondée sur l'âge et l'espérance de vie du patient et il n'est pas bien difficile de deviner le critère de sélection employé. La collectivité impose ici aux médecins la responsabilité d'un choix qu'elle ne veut pas assumer explicitement. La seule façon pour eux d'échapper à cette responsabilité serait d'informer systématiquement un patient auquel, pour des raisons de coût, ils ne peuvent proposer le traitement médical le mieux adapté à son cas.

Dans le cas de l'IVG, l'incitation est sociale et devient de plus en plus forte, comme le montre Danielle Moyse ${ }^{8}$ à propos de l'IVG en cas d'anomalie génétique, ou comme on peut le comprendre en analysant les mesures prises par Ségolène Royal à l'époque Ministre déléguée aux enseignements scolaires sur la contraception des élèves des collèges et lycées (BOEN, 6 janvier 2000) : les familles ont de moins en moins la parole dans ce genre de situations, au profit des infirmières scolaires. On néglige ici l'intérêt individuel et familial en le confiant à une personne qui n'est pas compétente pour l'apprécier et qui est soumise à une hiérarchie administrative.

Dans d'autres cas, l'obligation administrative est officielle, non seulement à l'hôpital, mais aussi en médecine privée : les références médicales opposables (RMO), décidées par une commission médicale, interdisent aux médecins libéraux de prescrire certains médicaments. Par exemple, la prescription simultanée de tranquillisant et de somnifère est interdite, et peut aboutir à une sanction contre un médecin enfreignant cette règle. Par suite, certains médecins sont amenés à la contourner en doublant les doses de tranquillisant et de somnifère et en les prescrivant alternativement.

La commission ne peut évidemment mieux évaluer l'état d'un malade qu'elle n'a jamais vu que le médecin traitant, et se rapporte en fait aux statistiques montrant une très importante consommation de tranquillisant en France par rapport aux autres pays : il s'agit bien d'une mesure collective indépendante de l'intérêt individuel.

Le danger est, comme l'écrit P.-H. Castel $^{9}$ :

Le triomphe de la médecine réductionniste sur la médecine holiste (qui prenait en compte l'individu malade comme totalité biopsychosociale), et le triomphe de la stratégie délibérée de liquidation de l'autorité clinique personnelle par le biais du traitement statistique des données médicales: objectivation radicale et abolition démocratique des écarts entre praticiens vont de pair.

\footnotetext{
${ }^{8}$ « Naissances coupables?», Esprit, $1^{\mathrm{er}}$ trimestre 2001.

9 «Des espèces nouvelles de l'hystérie collective ? L'exemple américain en débat », à paraître dans le Journal Français de Psychiatrie ou http://pierrehenri.castel.free.fr
} 
L'administration de la santé suit ainsi l'exemple de l'Éducation Nationale : la gestion d'un budget global fait que l'on substitue à la notion de santé individuelle, évaluée de façon subjective dans le cadre d'une relation individuelle entre le médecin et le patient, une notion de santé collective dont l'objectivité apparente peut se révéler contraire aux intérêts personnels.

\section{CONCLUSION}

L'utilisation de la statistique paraît actuellement abusive dans le processus de prise de décision à l'intérieur d'un système administratif chargé à la fois d'appliquer des réformes et d'en évaluer les résultats. En ce qui concerne l'éducation et la santé, cela paraît inquiétant.

Les experts en matière d'éducation et de santé ne sont plus les enseignants ni les médecins, mais les statisticiens, et les expertises consistent non pas à observer des cas individuels - ce que l'on appelle l'analyse qualitative en marketing - mais à mettre en place des modèles statistiques et à calculer des pourcentages vérifiant en réalité le suivi de la politique choisie.

Ces deux constatations s'expliquent simplement par le fait que la fonction d'utilité choisie dans les politiques éducatives et sanitaires ne coïncident pas du tout avec les utilités individuelles dont l'importance est évidemment fondamentale dans le cas des services publics.

Un des dangers principaux de cette démarche est de substituer à un ensemble d'erreurs individuelles une erreur collective, dont on connaît quelques exemples actuellement (scandales du sang contaminé, de l'encéphalopathie spongiforme bovine, de l'hormone de croissance, ...).

Un autre est l'apparition d'effets secondaires dans le comportement individuel, comme la déresponsabilisation des acteurs et l'augmentation d'inégalités sociales, et dans la société, comme le renforcement du contrôle social rendu possible par la codification systématique des comportements et des besoins.

On peut se demander si cette utilisation des statistiques ne témoigne pas d'une modification en profondeur de la démarche politique, consistant à définir les besoins individuels en fonction des objectifs politiques. 